SHORT REPORT

\title{
Coexistence of lactating adenoma and invasive ductal adenocarcinoma of the breast in a pregnant woman
}

\author{
A Saglam, B Can
}

J Clin Pathol 2005;58:87-89. doi: 10.1136/icp.2004.018275

\begin{abstract}
A 36 year old pregnant woman was admitted to hospital complaining of an enlarging mass in her left breast. Histopathological examination of the mastectomy specimen revealed a high grade infiltrating ductal adenocarcinoma intermixed with a lactating adenoma. Lactating adenomas are rare entities but are the most common masses that occur during pregnancy. Although they are not thought to carry an increased risk of cancer there are two other case reports in the literature of a lactating adenoma associated with an infiltrating carcinoma. In this case, areas where the lactating adenoma and the infiltrating carcinoma were intermixed could be identified. This case could simply be a collision tumour, although the possibility of an invasive carcinoma arising within a lactating adenoma cannot be ruled out. Because of the relative lack of experience with lactating adenomas, the question of an increased association with carcinoma development remains unclear.
\end{abstract}

B reast cancer diagnosed during pregnancy or 12 months postpartum is referred to as pregnancy associated breast cancer and is reported in 1/3000 pregnancies. ${ }^{1}$

Lactating adenomas are the most prevalent breast masses seen in pregnant women. ${ }^{2}$ Although they are not thought to carry an increased risk of cancer, Hertel et al reported the case of a patient who developed invasive ductal adenocarcinoma in the previous excision site of a lactating adenoma, and Geschicker and Lewis reported a lactating adenoma containing an associated infiltrating carcinoma. ${ }^{3}{ }^{4}$ We report the case of a pregnant woman with coexistent lactating adenoma and invasive ductal adenocarcinoma as components of the same mass. Considering the fact that lactating adenomas are rare and studies involving large series are lacking, we believe that such cases question whether lactating adenomas may be associated with an increased risk of cancer.

\section{CLINICAL SUMMARY}

A 36 year old pregnant woman was admitted to our hospital complaining of an enlarging mass in her left breast. According to her last menstrual period she was 21 weeks into her pregnancy and obstetrical ultrasonography confirmed a 22 week gestation. She had first noticed a lump in her left breast a year ago while she was still nursing her now 18 month old infant. After becoming pregnant again she realised that the lump was growing. Incisional biopsy at another institute revealed invasive adenocarcinoma and the patient was referred to our hospital.

The patient underwent modified radical mastectomy. She is now receiving chemotherapy and last month gave birth to a healthy baby.

\section{PATHOLOGICAL FINDINGS}

The mastectomy specimen measured $18 \times 15 \times 7 \mathrm{~cm}$. Serial sectioning showed a circumscribed tan/brown mass, $7 \mathrm{~cm}$ in diameter, which resembled the cut surface of a salivary gland, with lobulated architecture. However, at the superior border of this mass the lobular architecture was erased by a fibrotic grey/white homogeneous lesion with stellate borders (fig lA). This homogeneous area measured $9 \mathrm{~cm}$ in diameter, continuing throughout the superior border of the lobulated mass, and infiltrating the neighbouring breast tissue.

Microscopically, the well circumscribed tan/brown mass turned out to be a lactating adenoma characterised by a proliferation of benign ducts separated by sparse intervening stroma, with preservation of lobular architecture. The ducts were lined by vacuolated secretory cells and contained eosinophilic secretions within the lumens (fig lB).

However, the stellate homogeneous area at the superior border of this lactating adenoma turned out to be an invasive adenocarcinoma with very little tubule formation, pronounced pleomorphism, and high mitotic activity (fig 1C). It was considered to be grade III/III according to the modified Bloom Richardson criteria. The tumour displayed mixed morphology, with areas of classic high grade ductal adenocarcinoma, areas characterised by targetoid and single file growth pattern, and areas where infiltrating tumour cells could be identified within lakes of extracellular mucin (fig 1D). Interestingly, areas where the infiltrative carcinoma and the lactating adenoma were intermingled could be identified. In these areas, the infiltrating tumour had a vaguely nodular pattern reminiscent of the lobular architecture of the lactating adenoma, and showed continuity with the lobules of the lactating adenoma (fig 2A). Lobules harbouring both tubular adenoma and infiltrative carcinoma could be identified (fig 2B). Furthermore, there were areas within the lobules of the adenoma characterised by acini lined by dysplastic cells side by side with normal acini (fig 2C). Foci of high grade ductal carcinoma in situ were present both within and surrounding the infiltrative carcinoma, and in between the lobules of the lactating adenoma (fig 2D). All observed lymph nodes were metastatic.

Immunohistochemical studies revealed progesterone receptor immunoreactivity in less than $5 \%$ of the neoplastic cells. Oestrogen receptor immunoreactivity was lacking and $2+$ membranous staining was seen for c-erbB2. Immunoreactivity for these antigens was rare in the cells of the adenoma component.

\section{DISCUSSION}

Pregnancy associated breast cancer, although rare, is one of the most common cancers seen in pregnant women. ${ }^{5}$ As in our case, these are large, high grade tumours with lymph node metastasis, low frequency of hormone receptors, and high expression of cerbB2 and they have a poor prognosis. ${ }^{1}$ The most probable explanation for presentation at advanced stages of disease is that the symptoms are neglected; both the patients themselves and physicians tend to relate the symptoms to pregnancy associated changes, resulting in a delay in treatment. ${ }^{5}$ This may also be true for our patient, who had a tumour $9 \mathrm{~cm}$ in diameter at diagnosis-the lump 

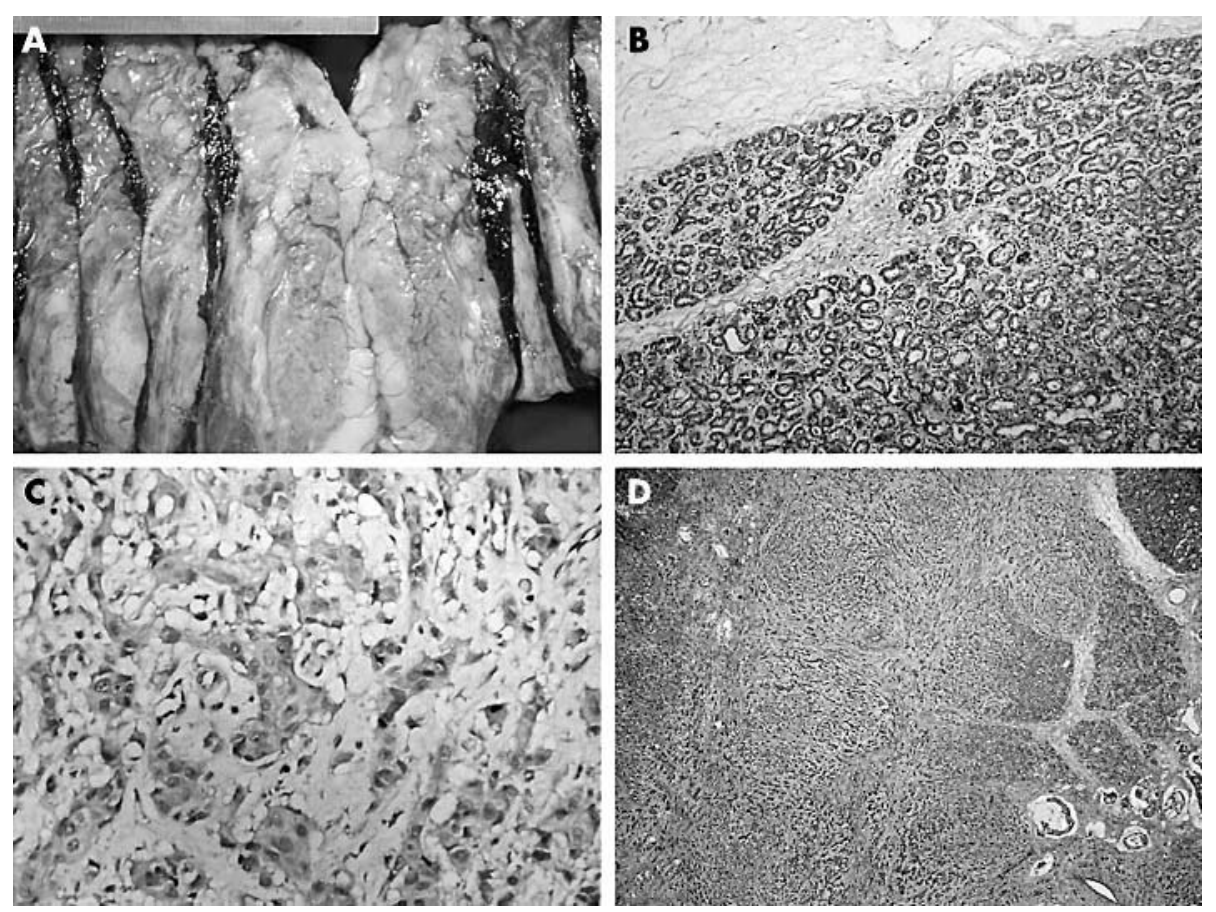

Figure 1 (A) Gross photograph showing cream coloured tumour at the top (the central cystic haemorrhagic area is the result of a previous biopsy) merging with a lactating adenoma at the bottom. (B) The lactating adenoma is well circumscribed and characterised by ducts lined with vacuolated secretory cells. (C) High power magnification of the infiltrating adenocarcinoma demonstrating its high grade nature. (D) Low power magnification showing an invasive carcinoma displaying mixed morphology infiltrating between the lobules of the lactating adenoma.

she had interpreted as lactational changes a year ago may have been the tumour itself. Alternatively, given the reported doubling time of breast tumours with high mitotic activity (which can be as short as 79 days), and considering that the tumour growth rate is higher in younger patients, ${ }^{6}$ it is also conceivable that the lump she had palpated was indeed the lactating adenoma, and that the tumour was well below the clinically detectable size at the time, or had not yet evolved, reaching its present size in just one year.

Lactating adenomas too are rare entities, but are the most common masses occurring during pregnancy. ${ }^{2}$ They are usually diagnosed by fine needle aspiration and regress spontaneously so that they are not often examined by biopsy. ${ }^{7}$ The aetiology of lactating adenomas remains unclear.
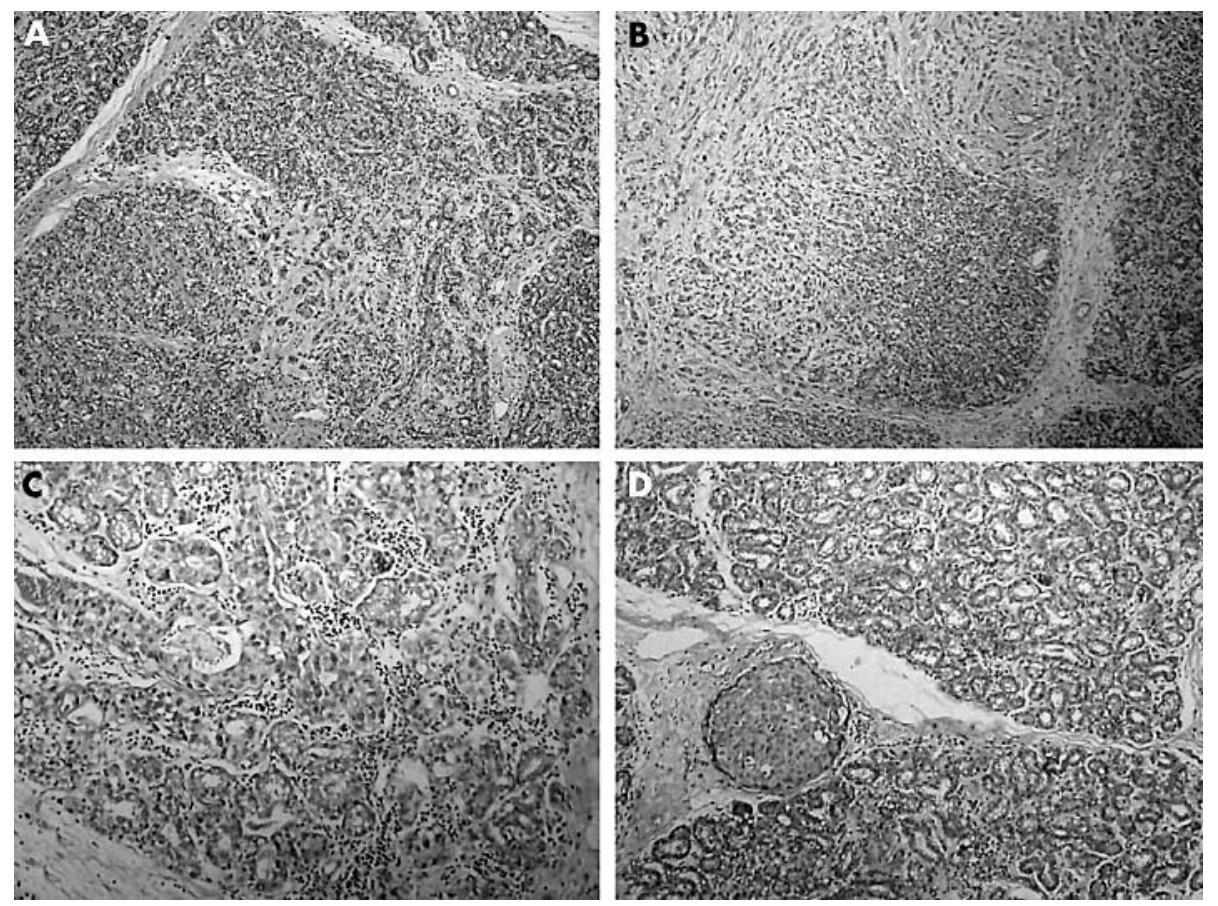

Figure 2 (A) Invasive carcinoma infiltrating within the lactating adenoma. (B) Lobules harbouring both tubular adenoma and infiltrative carcinoma. (C) Lobular cancerisation. (D) Foci of high grade ductal carcinoma in situ between the lobules of the lactating adenoma. 
Whether lactating adenomas represent tubular adenomas with varying histology as a result of hormonal changes brought about by pregnancy and lactation, or whether they are distinct entities of their own is also unknown. ${ }^{89}$ The consensus at this time is that lactating adenomas are tubular adenomas with lactational changes. ${ }^{2}{ }^{8}$

\section{"Recent evidence suggests that prolactin too has a role in human breast cancer"}

Lactating adenomas are benign lesions and are not thought to be a risk factor for the development of carcinoma. However, there is a report of a lactating adenoma occurring simultaneously with an infiltrative carcinoma and a case report of an invasive carcinoma developing at the previous excision site of a lactating adenoma. ${ }^{3}{ }^{4}$ These cases question the possibility of malignant transformation of lactating adenomas. In our case, areas of transition between the adenoma and the adenocarcinoma could be identified. Whether these areas of transition represent transformation of an adenoma to an adenocarcinoma or infiltration of the adenoma by the adjacent adenocarcinoma is uncertain. Our case could simply be a collision tumour, although one would expect complete effacement of the lactating adenoma component in a collision tumour. In our case, there were intact lobules of lactating adenoma within the infiltrative carcinoma, lobules harbouring both adenomatous and carcinomatous areas were present, carcinoma in situ was identified in between the lobules of the lactating adenoma, and there was lobular cancerisation within the lactating adenoma component. We believe all these factors are in favour of an infiltrative carcinoma developing in the setting of and possibly from a lactating adenoma.

The role of hormones in breast cancer development and regulation of breast cancer cell growth is very complex and remains an area of intense study. We know that during pregnancy high concentrations of oestrogen, progesterone, and prolactin promote the growth of ducts and the formation of tubuloalveolar structures in preparation for lactation. ${ }^{10}$ Progesterone and prolactin are especially known for their synergistic proliferative activity during pregnancy. Oestrogen and progesterone have long been known to play a well defined role in human breast cancer. Recent evidence suggests that prolactin too has a role in human breast cancer. ${ }^{11}{ }^{12}$ Our patient was breastfeeding when she became pregnant again and therefore already had high concentrations of prolactin. Lactating adenomas have been shown to express high amounts of the prolactin receptor, ${ }^{13}$ and excess prolactin has been shown to induce breast cancer in mice ${ }^{14}$; excess prolactin may have been present in our patient. The effects of high concentrations of prolactin and progesterone on an already fully primed breast (as a result of lactation) could have promoted rapid growth of an existing small focus of breast cancer cells. It is also plausible that this hormonal environment composed of excess prolactin, together with the effects of progesterone, could have initiated carcinogenesis in the lactating adenoma.

Fibroadenomas are known to increase the risk of breast carcinoma. $^{15}$ Furthermore, there are cases of carcinoma arising within tubular adenomas in the literature. ${ }^{16}{ }^{17} \mathrm{We}$ cannot rule out the possibility of an invasive carcinoma arising within a lactating adenoma in our case, perhaps brought about by the fluctuation of the hormonal milieu our patient experienced when she became pregnant while she was still nursing. In view of the fact that tubular adenomas and lactating adenomas are closely related, and may be variants of the same entity, our case and that reported by Hertel et al and Geschicker and Lewis may suggest that
Take home messages

- We report the case of a pregnant woman with a high grade infiltrating ductal adenocarcinoma intermixed with a lactating adenoma

- Although lactating adenomas are not thought to be risk factors for the development of carcinoma, the histological characteristics of this case suggest that the infiltrative carcinoma may have developed from the lactating adenoma, perhaps as a result of the hormonal environment composed of excess prolactin, together with the effects of progesterone

- Further experience is necessary to resolve this dilemma and determine its prognostic relevance

- Women with lactating adenomas should be followed up closely to rule out coexistent carcinoma, even if the chance is very remote

lactating adenomas too can be associated with an increased risk of cancer on certain occasions. Clearly, further experience is necessary to resolve the dilemma and determine its prognostic relevance. Until then, our case once again reminds us that women with lactating adenomas should not be neglected and close follow up should be maintained to rule out coexistent carcinoma, even if the chance is very remote.

\section{Authors' affiliations}

A Saglam, B Can, Department of Pathology, Hacettepe University Medical School, Sihhiye, Ankara, 06100, Turkey

Correspondence to: Dr A Saglam, Department of Pathology, Hacettepe University Medical School, Sihhıye, Ankara, 06100, Turkey; eminearzusaglam@yahoo.com

Accepted for publication 23 August 2004

\section{REFERENCES}

1 Reed W, Hannisdal E, Skovlund E, et al. Pregnancy and breast cancer: a population-based study. Virchows Arch 2003;443:44-50.

2 Baker TP, Lenert JT, Parker J, et al. Lactating adenoma: a diagnosis of exclusion. Breast J 2001;7:354-7.

3 Hertel BF, Zaloudek C, Kempson RL. Breast adenomas. Cancer 1976;37:2891-905

4 Geschicker CF, Lewis D. Pregnancy and lactation changes in fibro-adenoma of the breast. BMJ 1938;1:499-503.

5 Lishner M. Cancer in pregnancy. Ann Oncol 2003;14(suppl 3):iii31-6.

6 Brekelmans CT, van Gorp JM, Peeters PH, et al. Histopathology and growth rate of interval breast carcinoma. Cancer 1996;78:1220-8.

7 Grenko RT, Lee KP, Lee KR. Fine needle aspiration cytology of lactating adenoma of the breast. A comparative light microscopic and morphometric study. Acta Cytol 1990;34:21-6.

8 Lactating adenoma of the breast [editorial]. Lancet 1989;1:252.

9 Slavin JL, Billson VR, Ostor AG. Nodular breast lesions during pregnancy and lactation. Histopathology 1993;22:481-5.

10 Nass SJ, Davidson NE. The biology of breast cancer. Hematol Oncol Clin North Am 1999;13:311-32.

11 Vonderhaar BK. Prolactin involvement in breast cancer. Endocr Relat Cancer 1999;6:389-404.

12 Vonderhaar BK. Prolactin: the forgotten hormone of human breast cancer. Pharmacol Ther 1998;79:169-78.

13 Gill S, Peston D, Vonderhaar BK, et al. Expression of prolactin receptors in normal, benign, and malignant breast tissue: an immunohistological study. J Clin Pathol 2001;54:956-60.

14 Wennbo H, Gebre-Medhin M, Gritli-Linde A, et al. Activation of prolactin receptor but not the growth hormone receptor is important for induction of mammary tumors in transgenic mice. J Clin Invest 1997;100:2744-51.

15 Dupont WD, Page DL, Parl FF, et al. Long-term risk of breast cancer in women with fibroadenoma. N Engl J'Med 1994;331:10-15.

16 Hill RP, Miller FN. Adenomas of the breast. With case report of carcinomatous transformation in an adenoma. Cancer 1954;7:318-24.

17 Domoto H, Tsuda H, Miyakawa K, et al. Invasive ductal carcinoma associated with tubular adenoma of the breast. Pathol Int 2002;52:244-8. 